\title{
LncRNA SNHG5 promotes nasopharyngeal carcinoma progression by regulating miR- 1179/HMGB3 axis
}

\author{
Dengtao $\mathrm{Liu}^{1 \dagger}$, Yanpeng Wang ${ }^{2}$, Yigang Zhao ${ }^{2 \dagger}$ and Xiao Gu${ }^{2^{*}}$ (D)
}

\begin{abstract}
Background: Long noncoding RNAs (IncRNAs) have been reported to be important regulators in pathogenesis of human cancers, including nasopharyngeal carcinoma (NPC). Here, we mainly aimed to explore the mechanisms of LncRNA-SNHG5/ miR-1179/HMGB3 axis in NPC progression.

Methods: RT-qPCR and Western blot analysis were employed to detect mRNA and protein expressions. CCK-8, Transwell and dual luciferase reporter assays were applied to investigate functions of LncRNA-SNHG5/miR-1179/ HMGB3 axis.

Results: Upregulation of IncRNA-SNHG5 and downregulation of miR-1179 were identified in NPC, which were associated with adverse clinical outcomes. Functionally, upregulation of IncRNA-SNHG5 and downregulation of miR1179 accelerated NPC cell proliferation, migration and invasion. Furthermore, IncRNA-SNHG5 acted as a molecular sponge of miR-1179 in NPC. Besides that, upregulation of HMGB3 was found in NPC, and knockdown of HMGB3 restrained NPC progression. Moreover, HMGB3, a target of miR-1179, regulated NPC progression by mediating LncRNA-SNHG5/miR-1179 axis.
\end{abstract}

Conclusion: LnCRNA SNHG5 serves as a tumor promoter in NPC by sponging miR-1179 and upregulating HMGB3.

Keywords: SNHG5, Nasopharyngeal carcinoma, miR-1179, HMGB3

\section{Introduction}

Nasopharyngeal carcinoma (NPC) is one of the most common malignant tumors in China, and the incidence rate is the first of the malignant tumors of the ear, nose and throat [1]. The pathogenesis of NPC is multifaceted and is mainly affected by heredity, viral infection, and environment. Because NPC is sensitive to radiation therapy, radiation therapy is the treatment of choice for NPC patients. However, for advanced cancer and cases of recurrence after radiotherapy, surgical resection and chemical treatment are also indispensable means [2].

\footnotetext{
*Correspondence: baniuontb75006@163.com

${ }^{\dagger}$ Dengtao Liu and Yigang Zhao contributed equally to this work.

2E.N.T. Department, Linyi People's Hospital, No. 27 East section of Jiefang Road, Linyi 276000, Shandong Province, People's Republic of China Full list of author information is available at the end of the article
}

Moreover, due to the easy recurrence and early metastasis of tumors, the prognosis of patients with NPC is poor. The 5-year survival rate for patients who are not sensitive to radiation is about $10 \%$, and the radiationsensitive 5-year survival rate is about 30\% [3]. Therefore, it is necessary to explore effective targets to improve the survival rate of NPC patients.

Long noncoding RNAs (lncRNAs) have been demonstrated to serve as tumor promoter or inhibitor in the pathogenesis of human cancers. Furthermore, lncRNA SOX2-OT promoted proliferation and metastasis of NPC cells through miR-146b-5p/HNRNPA2B1 pathway [4]. On the contrary, IncRNA-LET was downregulated in NPC tissues and restrained proliferation and invasion of NPC cells [5]. Recently, the specific roles of lncRNA

(c) The Author(s). 2020 Open Access This article is licensed under a Creative Commons Attribution 4.0 International License, which permits use, sharing, adaptation, distribution and reproduction in any medium or format, as long as you give appropriate credit to the original author(s) and the source, provide a link to the Creative Commons licence, and indicate if changes were made. The images or other third party material in this article are included in the article's Creative Commons licence, unless indicated otherwise in a credit line to the material. If material is not included in the article's Creative Commons licence and your intended use is not permitted by statutory regulation or exceeds the permitted use, you will need to obtain permission directly from the copyright holder. To view a copy of this licence, visit http://creativecommons.org/licenses/by/4.0/ The Creative Commons Public Domain Dedication waiver (http://creativecommons.org/publicdomain/zero/1.0/) applies to the data made available in this article, unless otherwise stated in a credit line to the data. 
Small Nucleolar RNA Host Gene 5 (SNHG5) caught our attention. In particularly, upregulation of SNHG5 was identified in melanoma and glioma and function as an oncogene [6, 7]. But SNHG5 was found to be downregulated in gastric cancer and blocked gastric cancer progression by trapping MTA2 [8]. These findings imply that SNHG5 has tissue specificity. Moreover, the dysregulation of SNHG5 remains unknown in NPC. Thus, this study was designed to explain the regulatory mechanism of SNHG5 in NPC.

Increasing studies suggest that lncRNAs involve in tumorigenesis by acting as miRNA sponges [9]. Here, miR-1179 was predicted to have binding sites with SNHG5. Previous studies have shown that the expression level of miR-1179 depends on the type of cancer. For example, miR-1179 was upregulated in esophageal squamous cell carcinoma and promoted cell invasion [10]. In contrast, miR-1179 was downregulated in pancreatic cancer and acted as a tumor suppressor [11]. For $\mathrm{NPC}$, the role of miR-1179 is still unclear, which need to be elucidated. In addition, high mobility group box 3 (HMGB3) was predicted to be a target of miR-1179 in this study. Moreover, the dysregulation of HMGB3 has been found in many human cancers. For example, upregulation of HMGB3 was identified in gastric cancer, and knockdown of HMGB3 inhibited proliferation and migration of gastric cancer cells [12]. Moreover, overexpression of HMGB3 promoted cell proliferation, migration and was associated with poor prognosis in urinary bladder cancer patients [13]. Additionally, it has been reported that inhibition of HMGB3 induced by miR-205$5 p$ inhibited cancer cell aggressiveness and was involved in prostate cancer pathogenesis [14]. However, the relationship between miR-1179 and HMGB3 is unclear in NPC.

The purpose of this study was to preliminarily elucidate the molecular mechanism of SNHG5/miR-1179/ HMGB3 pathway in NPC progression. Furthermore, the functions of SNHG5, miR-1179 and HMGB3 were also investigated in NPC. Our results will provide potential targets for NPC treatments.

\section{Materials and methods}

\section{Clinical tissues}

Sixty-four NPC tissues and adjacent normal samples were obtained from patients in Linyi People's Hospital, who did not receive any treatments prior to surgery. Informed consents were obtained from all NPC patients. This study was approved by the Institutional Ethics Committee of Linyi People's Hospital.

\section{Cell culture}

Normal nasopharyngeal epithelial cells NP69 (BNCC338439) and NPC cell line C666-1
(BNCC337872) were purchased from BeNa Culture Collection (BNCC, Beijing, China) in February, 2018. NP69 and C666-1 cells are not misidentification and contamination of human cell lines (ExPASy: SIB Bioinformatics Resource Portal, https://www.expasy.org/). These cells were seeded in culture solution (90\%RPMI-1640+ $10 \% \mathrm{FBS}$ ) at $37^{\circ} \mathrm{C}$ in a humid atmosphere with $5 \% \mathrm{CO}_{2}$.

\section{Cell transfection}

SNHG5 complementary DNA was synthesized and cloned into the expression vector pcDNA3.1. Effective siRNA oligonucleotides targeting SNHG5 and HMGB3 or miR-1179 mimic, miR-1179 inhibitor was obtained from GenePharma (Shanghai, China). Next, they were transfected into C666-1 cells using Lipofectamine 2000 (Invitrogen/Thermo Fisher Scientifc), respectively. Untreated cells were set as the control (Blank).

\section{Real-time quantitative PCR (RT-qPCR)}

Total RNA was extracted using TRIzol reagent (Invitrogen, USA). RNA was reversely transcript into complementary DNA (cDNA) using PrimeScript RT reagent kit (Takara, Dalian, China). RT-qPCR assay was performing using Real-time PCR Mixture assays (Takara) on ABI 7300 Real-time PCR system (Applied Biosystems, Waltham, MA). SNHG5 and miR-1179 expression were normalized to U6, while HMGB3 was normalized to GAPDH. Their expressions were quantified with the $2^{-\triangle \Delta \mathrm{cq}}$ method.

\section{Western blot analysis}

RIPA lysis buffer (Beyotime) was used to obtain protein samples. Next, 10\% SDS-PAGE was used to separate protein. Protein samples were transferred to PVDF membranes. Blocked with 5\% non-fat milk, protein samples were incubated overnight at $4{ }^{\circ} \mathrm{C}$ with E-cadherin, $\mathrm{N}$-cadherin, $\mathrm{Bcl}-2$, Bax and GAPDH primary antibodies (Abcam, Shanghai, China). Afterwards, secondary antibodies (Abcam, USA) were added to incubate protein samples for $1 \mathrm{~h}$. ECL kit (Beyotime) was used to assess protein bands.

\section{Cell counting Kit-8 (CCK-8) assay}

Transfected C666-1 cells $\left(2 \times 10^{3}\right.$ cells/well $)$ were seeds in 96-well plates. Next, these cells were incubated for $24,48,72$ or $96 \mathrm{~h}$ in RPMI-1640 medium, respectively. Then, $10 \mu \mathrm{L}$ CCK- 8 reagents were used to incubate the cells for $4 \mathrm{~h}$. The medium was discarded and dimethyl sulfoxide was added. After 10 min shaking, OD490 was detected by a microplate reader (Olympus Corp., Tokyo, Japan). 


\section{Transwell assay}

Cell invasion was detected in the upper chamber with Matrigel. After $30 \mathrm{~min}$, Transwell upper chamber was added with C666-1 cell suspension $\left(2 \times 10^{3}\right.$ cells/well). Next, RPMI-1640 medium (10\% FBS) was added to 24well plates in lower chamber. After $24 \mathrm{~h}, 0.1 \%$ crystal violet was applied to stain the moved cells. Cell migration experiment was performed without Matrigel. Observation and photographing were performed by a light microscope.

\section{Flow cytometric analysis}

Flow cytometric analysis was adopted to detect apoptosis in NPC. First, transfected C666-1 cells $\left(3 \times 10^{3}\right.$ cell/well) were seed in 6-well plates. After $48 \mathrm{~h}$, trypsin (without EDTA) digestion was used to collect C666-1 cells. We then suspended the collected C666-1 cells in PBS at $4{ }^{\circ} \mathrm{C}$. After discarding PBS, Annexin V-FITC from Annexin V-FITC Apoptosis Detection Kit (Biovision, K101) was used to stain C666-1 cells for 15 mins following with Binding Buffer (1×). After that, C666-1 cells were stained with PI (Sigma-Aldrich, Shanghai, China). Finally, we observed the apoptosis of C666-1 cells using a flow cytometer (BD Biosciences).

\section{Immunocytochemical assay}

The section of NPC tissues were dewaxed, hydrated, and washed twice with PBS for $5 \mathrm{~min}$. After blocking with $5 \%$ goat serum (diluted in PBS), we incubated the cells with anti-HMGB3 antibody at $37^{\circ} \mathrm{C}$ for $1-2 \mathrm{~h}$. Then, we washed them for three times with PBS for $5 \mathrm{~min}$. Subsequently, we incubated them with the HRP-conjugated goat anti-rabbit secondary antibody at $37^{\circ} \mathrm{C}$ for $1 \mathrm{~h}$. After washing 3 times with PBS, DAB mixture was used for color development of this section. The section was washed, counterstained, dehydrated, transparentized and mounted. Images were captured using microscope.

\section{Dual luciferase reporter assay}

The 3'-UTR of wild-type and mutant SNHG5 (wtSNHG5 and mut-SNHG5) or HMGB3 (wt-HMGB3 and mut-HMGB3) were amplified and then inserted into pmiR-GLO vector (Promega Beijing Biotech Co., Beijing, China). Then, the above reporter plasmids were transfected into C666-1 cells with miR-1179 mimics. After $48 \mathrm{~h}$, activities of firefly luciferase were determined by dual-luciferase reporter assay system (Promega, USA).

\section{Statistical analysis}

Data were analyzed using Student's t-test or one-way ANOVA in SPSS 19.0 or Graphpad Prism 6. Data are shown as mean \pm SD. Univariate Kaplan-Meier method with log-rank test was used to analyze the association between SNHG5 and survival rate. $P<0.05$ was defined as statistically difference.

\section{Results \\ The dysregulation of LncRNA-SNHG5 and miR-1179 was found in NPC}

The abnormal expressions of LncRNA-SNHG5 and miR-1179 were detected in NPC tissues. First, LncRNASNHG5 expression was increased in NPC tissues compared to normal tissues (Fig. 1a). Similarly, upregulation of LncRNA-SNHG5 was also identified in C666-1 cells compared with NP69 cells (Fig. 1b). Additionally, higher LncRNA-SNHG5 expression was found in advanced stages than in early stages of NPC patients (Fig. 1c). And poor prognosis in NPC patients was related to high LncRNA-SNHG5 expression (Fig. 1d). Next, we found that miR-1179 expression was downregulated in NPC tissues contrast to normal tissues (Fig. 1e). Furthermore, miR-1179 was also decreased in C666-1 cells compared with NP69 cells (Fig. 1f). Moreover, miR-1179 was much lower in advanced stages of NPC patients than in early stages (Fig. 1g). Furthermore, downregulation of miR1179 was associated with shorter overall survival in NPC patients (Fig. 1h). Based on these results, we suspect that LncRNA-SNHG5 and miR-1179 may be affected the tumorigenesis of NPC.

LncRNA-SNHG5 acted as a molecular sponge of miR-1179 in NPC

Next, miR-1179 was predicted to have binding sites with LncRNA-SNHG5 in starBase version 2.0 (http://starbase. sysu.edu.cn/, Fig. 2a). Dual luciferase reporter was then performed to verify the above prediction. We found that miR-1179 mimics obviously reduced the luciferase activity of wt-SNHG5, but had little effect on mut-SNHG5 in C666-1 cells (Fig. 2b). Moreover, LncRNA-SNHG5 was found to inversely regulate miR-1179 expression in NPC tissues (Fig. 2c). Next, miR-1179 expression was observed in C666-1 cells with si-SNHG5 and SNHG5 vector. RT-qPCR showed that upregulation of SNHG5 decreased miR-1179 expression, while knockdown of SNHG5 increased miR-1179 expression (Fig. 2d). At the same time, how miR-1179 regulates LncRNA-SNHG5 expression was also detected in C666-1 cells. LncRNASNHG5 expression was also reduced by miR-1179 mimics and enhanced by miR-1179 inhibitor (Fig. 2e). These results imply that LncRNA-SNHG5 acts as a molecular sponge of miR-1179 in NPC.

\section{LncRNA-SNHG5/miR-1179 axis was involved in NPC progression}

To further assess the regulatory mechanism of LncRNASNHG5/miR-1179 in NPC, miR-1179 mimics, miR-1179 inhibitor, si-SNHG5 or SNHG5 vector was transfected 
A

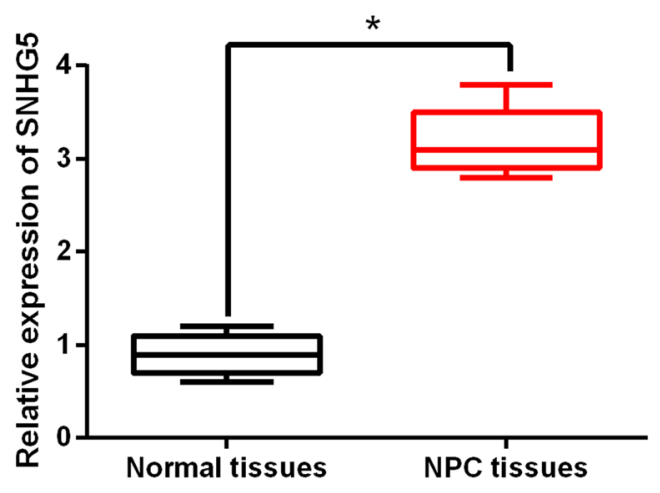

C

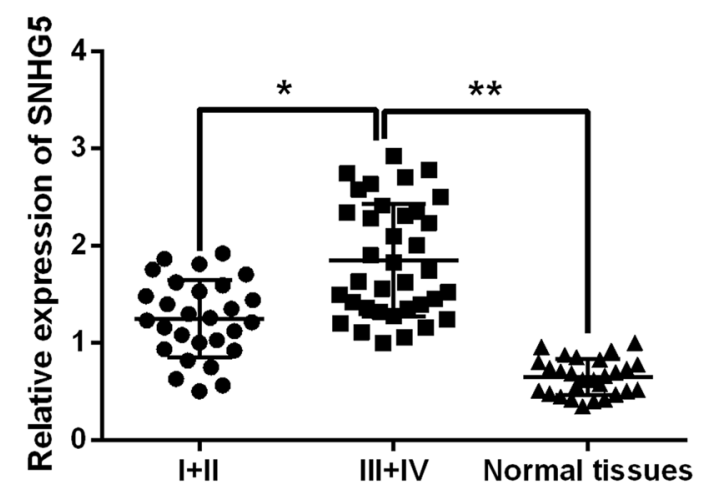

E

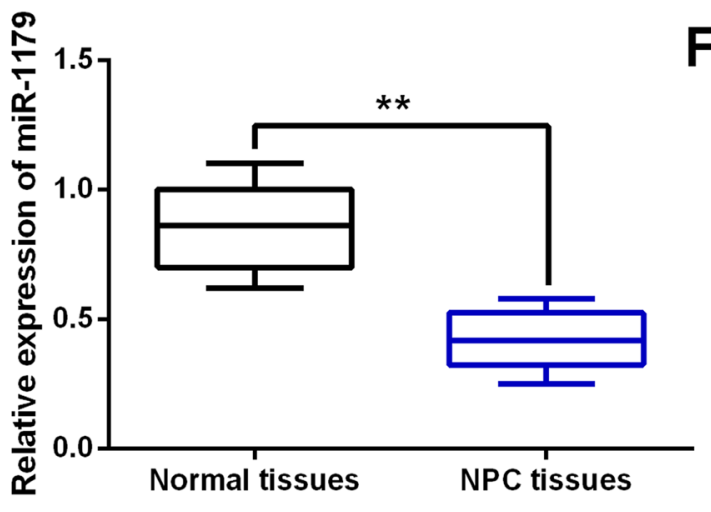

B

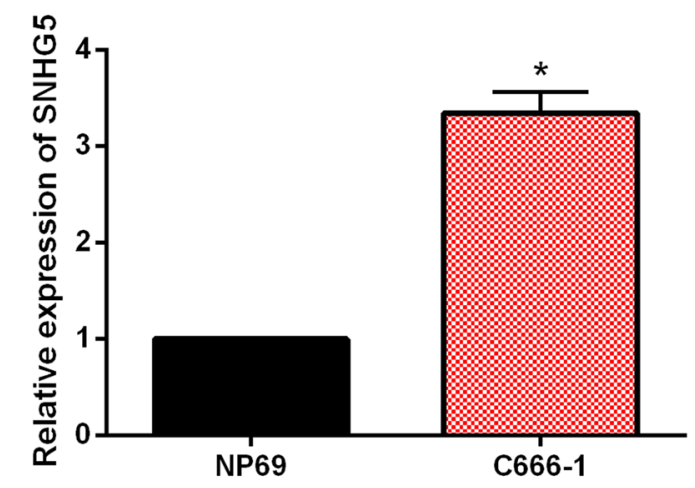

D

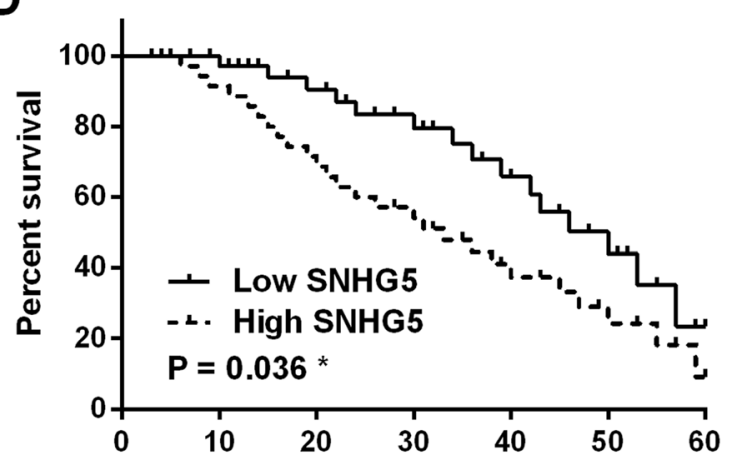

F 2 Time (months)

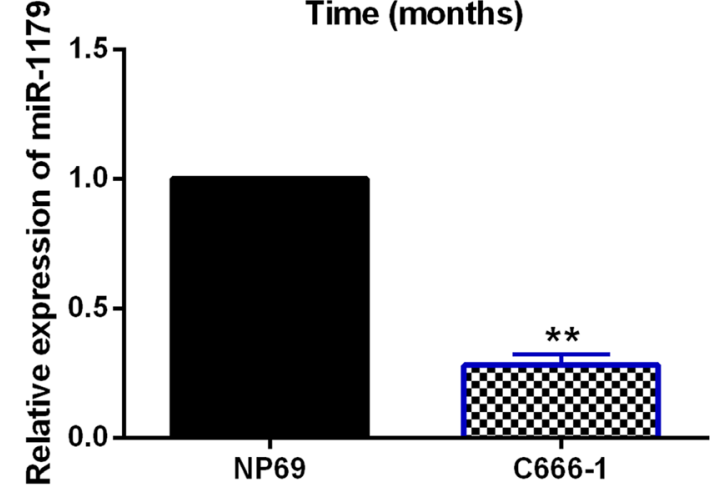

G

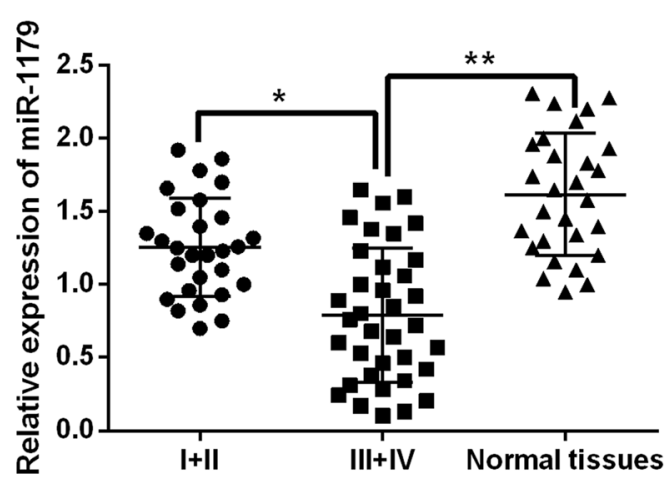

H

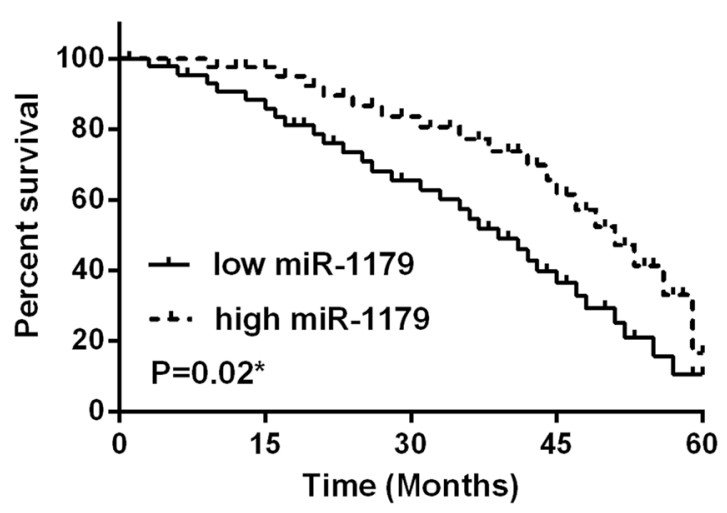


(See figure on previous page.)

Fig. 1 The dysregulation of LncRNA-SNHG5 and miR-1179 was found in NPC. a The LncRNA-SNHG5 expression in NPC tissues and normal tissues b LncRNA-SNHG5 expression in C666-1 and NP69 cells c LnCRNA-SNHG5 expression in different clinical stage of NPC patients $\mathbf{d}$ High LncRNASNHG5 expression patients predicted worse prognosis. e The miR-1179 expression in NPC tissues and normal tissues $\mathbf{f}$ MiR-1179 expression in C666-1 and NP69 cells $\mathbf{g}$ MiR-1179 expression in different clinical stage of NPC patients $\mathbf{h}$ Low miR-1179 expression patients predicted worse prognosis. ${ }^{* *} P<0.05,{ }^{* *} P<0.01$

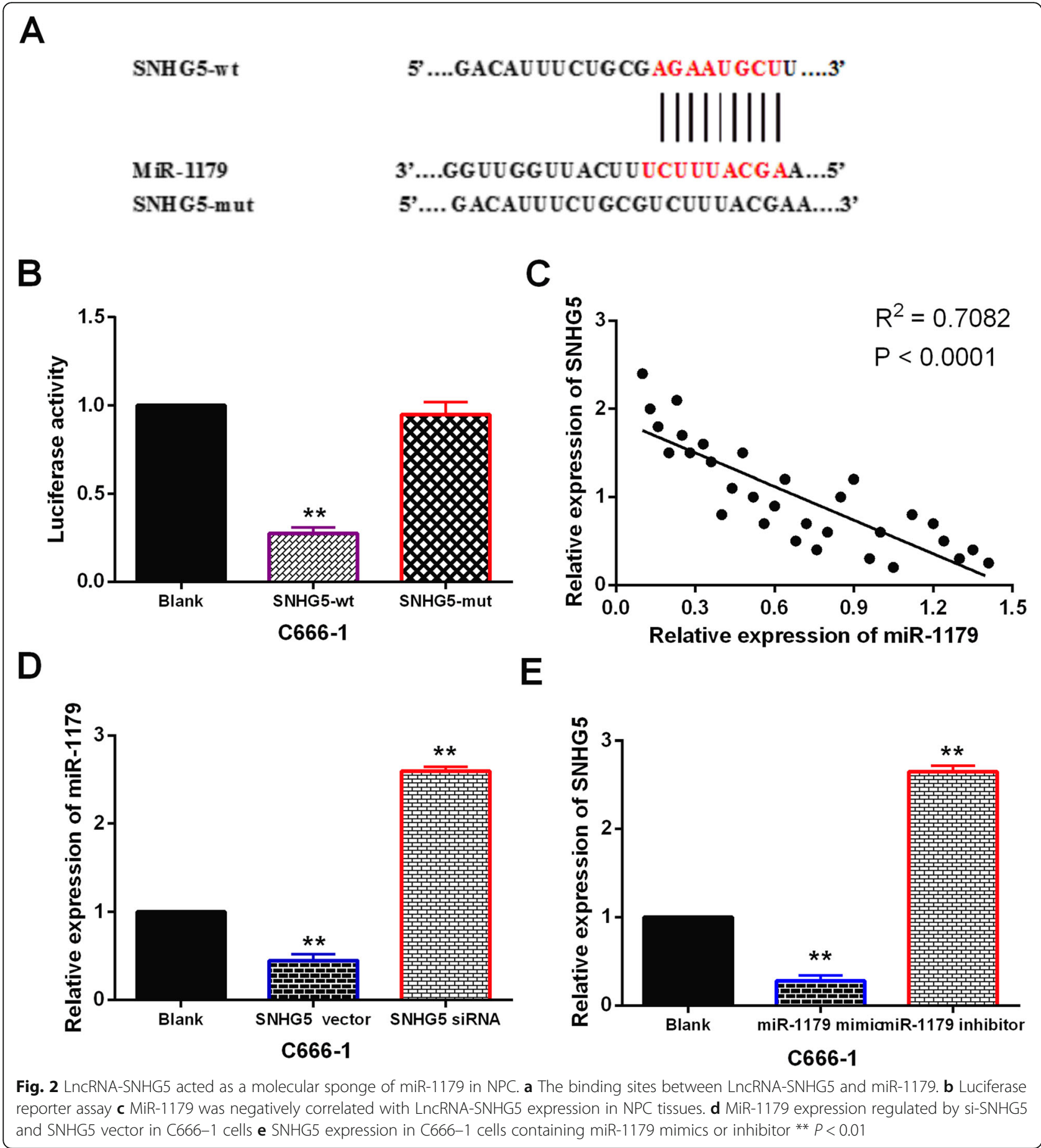




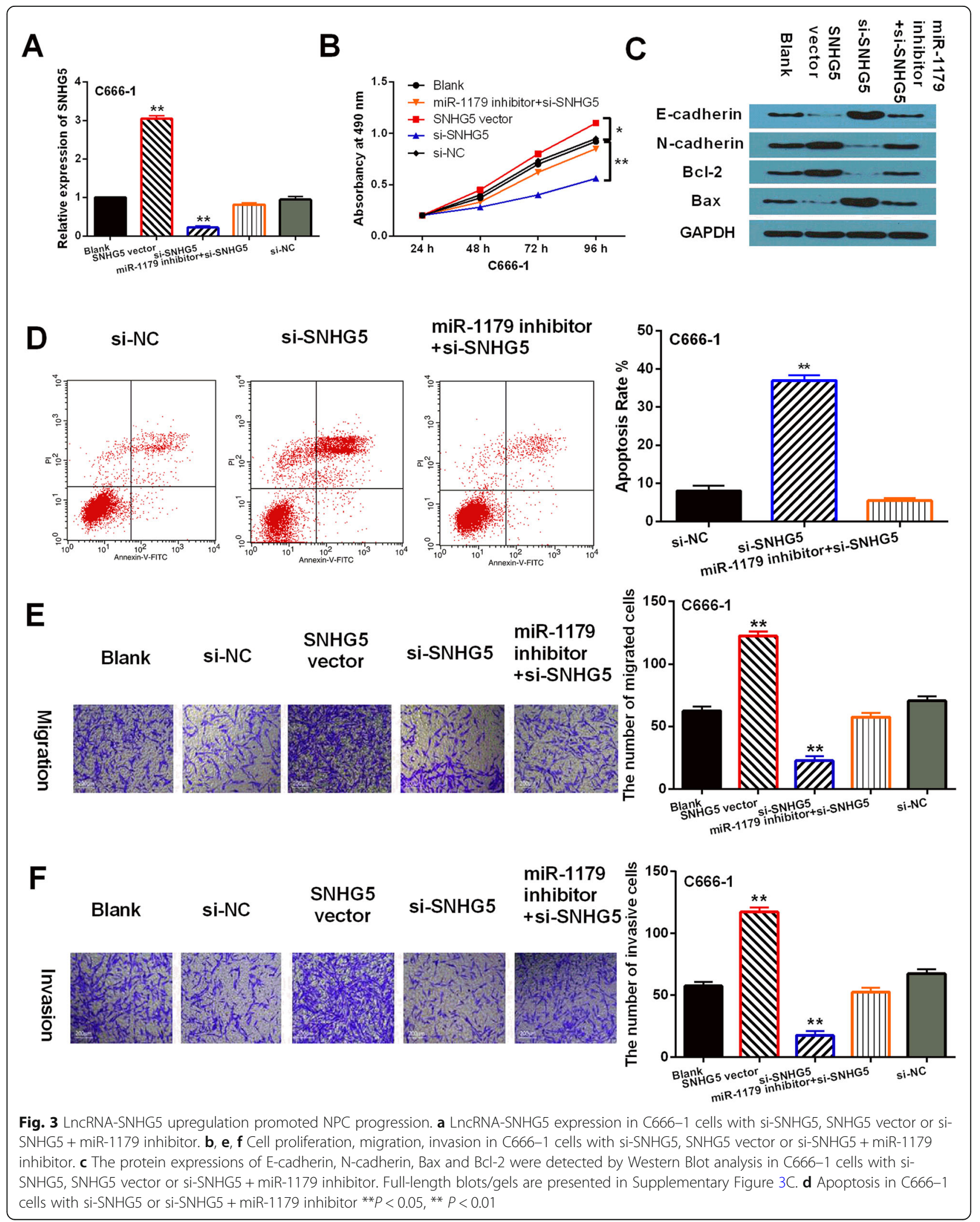


into C666-1 cells, respectively. RT-qPCR indicated that LncRNA-SNHG5 expression was downregulated by siSNHG5 and upregulated by SNHG5 vector in C666-1 cells. After transfection of miR-1179 inhibitor, the decreased expression of LncRNA-SNHG5 induced by siSNHG5 was recovered (Fig. 3a). Functionally, CCK-8 assay suggested that upregulation of LncRNA-SNHG5 promoted cell proliferation, while knockdown of LncRNA-SNHG5 restrained proliferation of C666-1 cells. And miR-1179 inhibitor abolished the inhibitory effect of si-SNHG5 on C666-1 cell proliferation (Fig. 3b). Additionally, downregulation of LncRNA-SNHG5 promoted E-cadherin and Bax expression and inhibited Ncadherin and $\mathrm{Bcl}-2$ expressions, while LncRNA-SNHG5 overexpression showed opposite effect on these genes in C666-1 cells. Similarly, miR-1179 inhibitor also exerted reverse effect on these genes regulated by si-SNHG5
(Fig. 3c). We also found that knockdown of LncRNASNHG5 induced apoptosis of C666-1 cells. Furthermore, miR-1179 inhibitor abolished the effect of LncRNA-SNHG5 on apoptosis in C666-1 cells (Fig. 3d). In addition, Transwell assay showed that cell migration and invasion were promoted by SNHG5 vector and restrained by si-SNHG5. Furthermore, si-SNHG5 mediated inhibition of cell migration and invasion was also restored by miR-1179 inhibitor (Fig. 3e, f). These results reveal that LncRNA-SNHG5 serves as a tumor promoter in NPC by targeting miR-1179.

Besides that, we found that miR-1179 mimics declined its expression, whereas miR-1179 inhibitor enhanced its expression in C666-1 cells. However, SNHG5 vector reduced the increased expression of miR-1179 induced by its mimics (Fig. 4a). Functionally, cell proliferation, migration and invasion were restrained by miR-1179

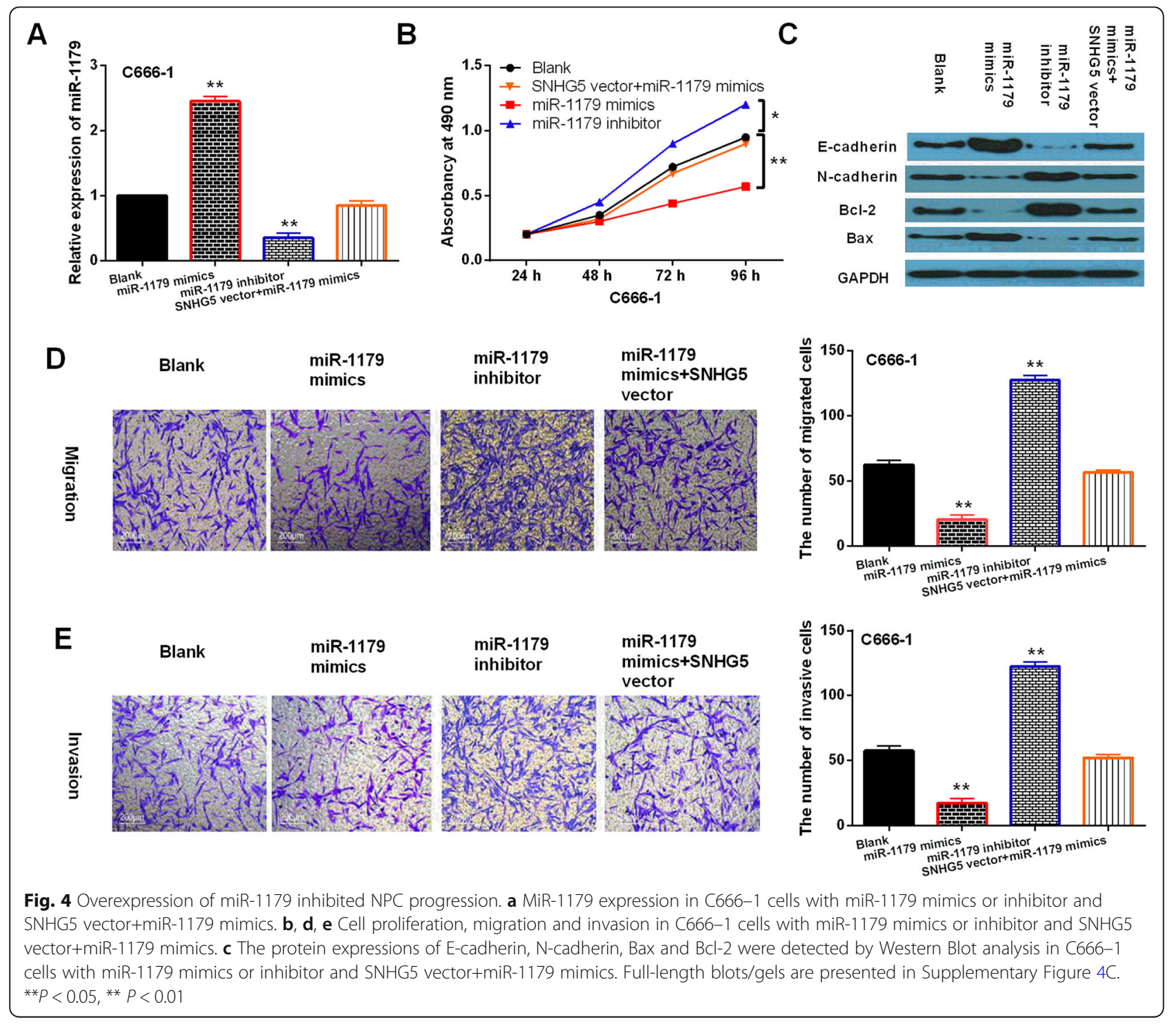


overexpression and accelerated by downregulation of miR-1179. And the reverse effect of SNHG5 vector on miR-1179 mimics mediated inhibition of cell proliferation, migration and invasion was also found in C666-1 cells (Fig. 4b, d, e). Additionally, miR-1179 mimics was found to promote E-cadherin and Bax expression and restrain $\mathrm{N}$-cadherin and Bcl-2 expressions, while miR1179 inhibitor exerted opposite effect. Similarly, SNHG5 vector also exerted reverse effect on these genes regulated by miR-1179 mimics in C666-1 cells (Fig. 4c). The results suggest that miR-1179 functions as a tumor inhibitor in NPC by interacting with LncRNA-SNHG5.

\section{HMGB3 is a direct target of miR-1179}

MiRNAs are well-known to involve in tumorigenesis by binding to target genes. TargetScan (http://www.targetscan.org) predicted that miR-1179 has binding sites with the 3'-UTR of HMGB3 (Fig. 5a). Luciferase reporter assay indicated that miR-1179 mimics declined wtHMGB3 luciferase activity, but had no effect on mutHMGB3 (Fig. 5b). At the same time, we found that HMGB3 expression was downregulated by miR-1179 mimics and upregulated by miR-1179 inhibitor (Fig. 5c). Moreover, HMGB3 was found to be upregulated in NPC tissues compared to normal tissues (Fig. 5d). The results of IHC displayed positive detection of HMGB3 protein expressions in the nucleus of NPC tissue (Fig. 5e). Moreover, the protein expression intensity of HMGB3 was obviously increased in NPC tissues compared to the adjacent normal tissues (Fig. 5f). And higher HMGB3 expression was identified in advanced stages of NPC patients than in early stages (Fig. 5g). Additionally, a negative correlation between HMGB3 and miR-1179 expressions was identified in NPC tissues (Fig 5h). But LncRNA-SNHG5 was found to positively regulate HMGB3 expression in NPC tissues (Fig. 5i). These

A

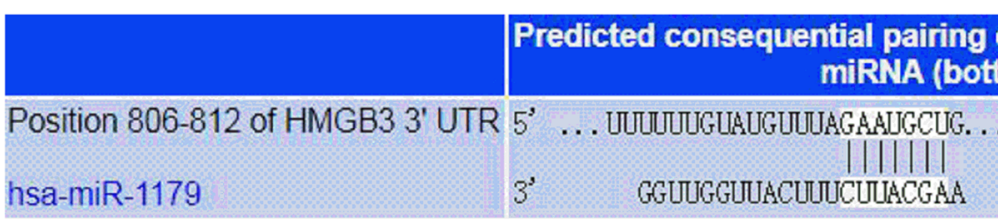

\section{B}

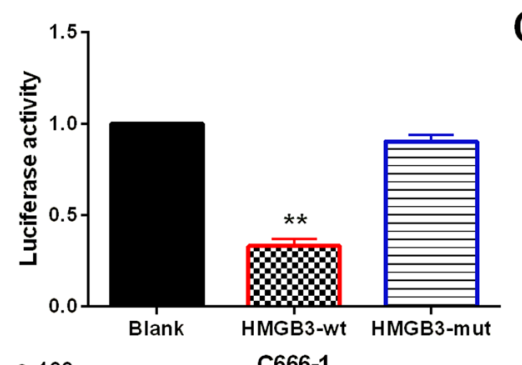

$\mathbf{F}$

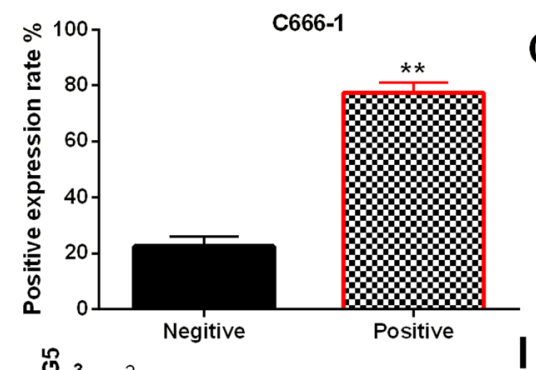

$\mathrm{H}$

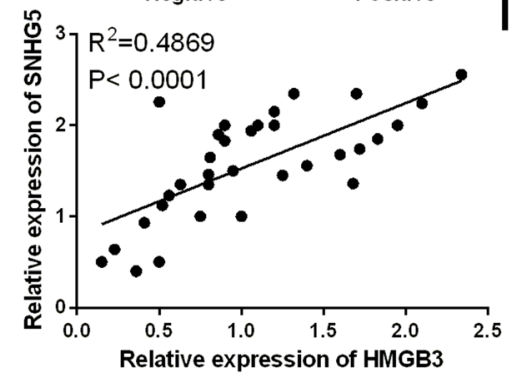

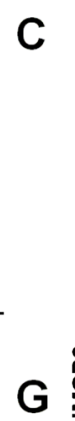
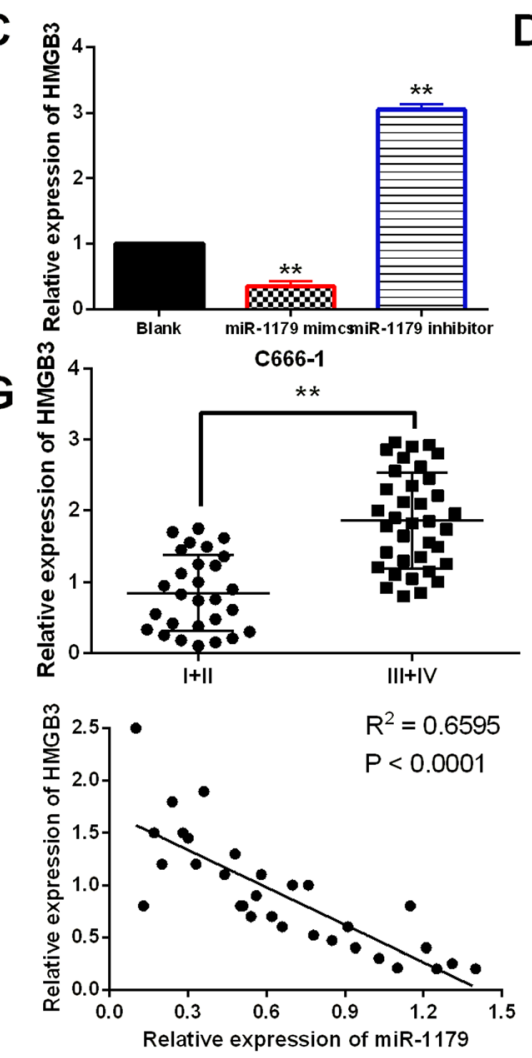

D

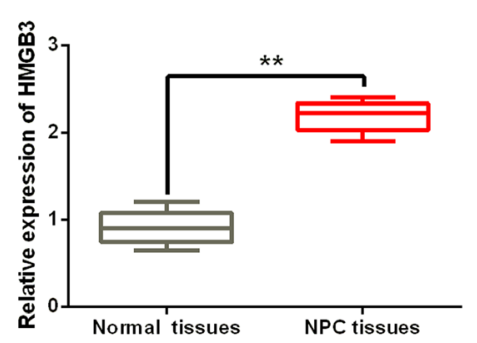

E Negitive

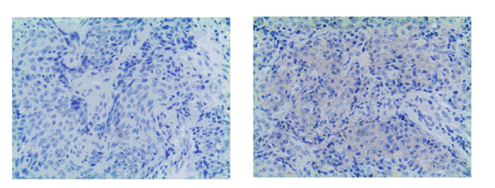

Positive

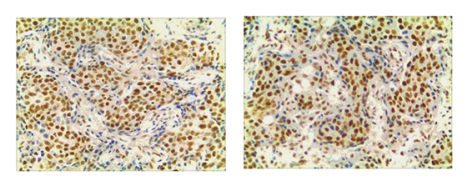

Fig. 5 HMGB3 is a direct target of miR-1179. a The binding sites between HMGB3 and miR-1179. b Luciferase reporter assay c HMGB3 expression regulated by miR-1179 mimics or inhibitor in C666-1 cells $\mathbf{d}$ HMGB3 expression in NPC tissues and normal tissues. $\mathbf{e}$, $\mathbf{f}$ The protein expression of HMGB3 in NPC tissues detected by immunohistochemistry. $\mathbf{g}$ HMGB3 expression in different clinical stage of NPC patients. $\mathbf{h}$ SNHG5 was positively correlated with HMGB3 expression in NPC tissues. i HMGB3 was negatively correlated with miR-1179 expression in NPC tissues. ${ }^{*} P<0.01$ 
results indicated that HMGB3 is a direct target of miR1179.

HMGB3 regulated NPC progression by mediating LncRNASNHG5/miR-1179 axis

To further explore the interaction between HMGB3 and LncRNA-SNHG5/miR-1179 axis, SNHG5 vector or miR-1179 inhibitor was transfected into C666-1 cells with si-HMGB3. First, we found that si-HMGB3 significantly reduced its expression in C666-1 cells. But SNHG5 vector or miR-1179 inhibitor recovered this reduction of HMGB3 (Fig. 6a). Functionally, knockdown of HMGB3 restrained proliferation of C666-1 cells, while SNHG5 vector or miR-1179 inhibitor recovered siHMGB3 mediated inhibition of cell proliferation (Fig. 6b). Moreover, knockdown of HMGB3 was found to promote E-cadherin and Bax expression and restrain $\mathrm{N}$-cadherin and Bcl-2 expressions in C666-1 cells. SNHG5 vector or miR-1179 inhibitor also reversely regulated the effect of si-HMGB3 on these genes (Fig. 6c). In addition, cell migration and invasion were found to be restrained by downregulation of HMGB3. And SNHG5 vector or miR-1179 inhibitor abolished the suppressive effect of si-HMGB3 on C666-1 cell migration and invasion (Fig. 6d, e). Collectively, LncRNASNHG5/miR-1179 axis promoted NPC progression by regulating HMGB3 expression.

\section{Discussion}

Recently, many lncRNAs have been reported to involve in tumorigenesis of NPC. For example, LncRNALINC00460 facilitated NPC tumorigenesis through sponging miR-149-5p to up-regulate IL6 [15]. In this study, LncRNA-SNHG5 was found to promote NPC progression by mediating miR-1179/HMGB3 axis. Specifically, upregulation of lncRNA-SNHG5 was identified in NPC, which was associated with clinical stages and poor prognosis. Functionally, upregulation of lncRNA-SNHG5 was

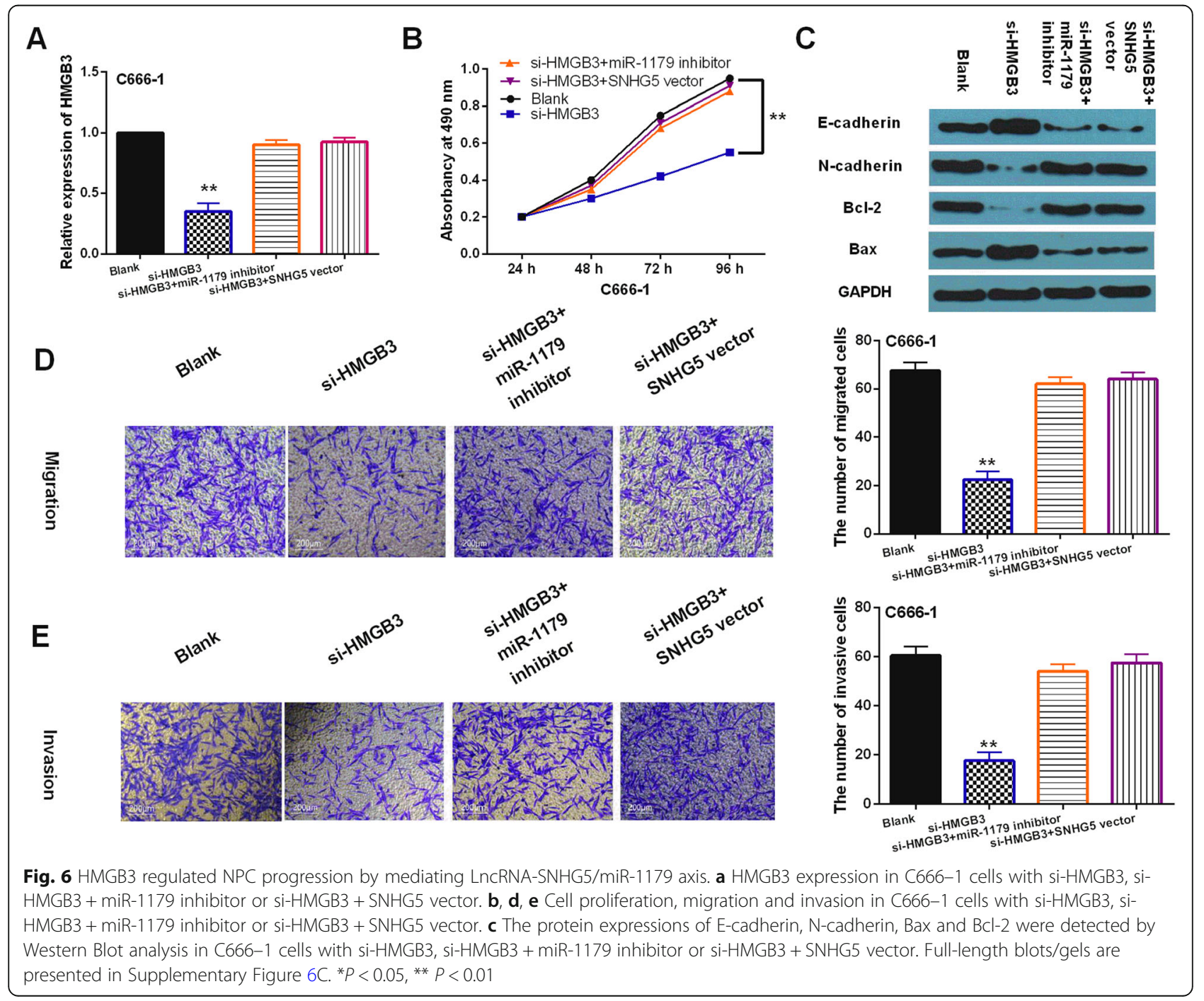


found to accelerate proliferation, migration and invasion of NPC cells. Consistent with our results, it was reported that IncRNA-SNHG5 was up-regulated and served as a prognostic biomarker in acute myeloid leukemia [16]. Moreover, lncRNA-SNHG5 has been demonstrated to facilitate osteosarcoma cell proliferation, migration and invasion [17], which was the same as our results. These findings imply that lncRNA-SNHG5 was upregulated in NPC and serves as a tumor promoter in NPC progression.

Many studies have demonstrated that lncRNAs function as miRNA sponges to participate in tumorigenesis. LncRNA-SNHG5 has been found to promote the development of colorectal cancer by sponging miR-132-3p/ CREB5 [18]. In our research, IncRNA-SNHG5 was confirmed as a molecular sponge of miR-1179. In addition, miR-1179 was downregulated in NPC and associated with adverse clinical outcomes in NPC patients. Furthermore, overexpression of miR-1179 inhibited NPC cell proliferation, migration and invasion. Similarly to our results, miR-1179 expression was decreased and restrained glioblastoma cell proliferation and cell cycle progression [19]. And miR-1179 was also found to suppress cell growth and invasion by targeting SPAG5 in human nonsmall cell lung cancer [20]. Besides that, the interaction between lncRNA-SNHG5 and miR-1179 was identified in NPC progression. LncRNA-SNHG5 was found to promote NPC development by sponging miR-1179, which has not been reported in previous studies. All these results demonstrate that miR-1179 acts as a tumor inhibitor in NPC.

Next, we found that HMGB3 is a direct target of miR1179. The dysregulation of HMGB3 has been identified in many human cancers. For example, increased expression of HMGB3 was found in esophageal squamous cell carcinoma, which predicted worse outcome [21]. Additionally, HMGB3 was found to be upregulated in glioblastoma and promoted cell proliferation and metastasis [22]. Here, upregulation of HMGB3 was identified in NPC and associated with advanced tumor stage in NPC patients. Furthermore, knockdown of HMGB3 suppressed NPC development. Our results are consistent with the above findings. Based on these findings, HMGB3 was confirmed to act as an oncogene in NPC. Besides that, we found miR-1179 inhibited NPC progression by downregulating HMGB3. Similarly, miR-758 was reported to inhibit proliferation, migration, invasion of non-small cell lung cancer cells by negatively regulating HMGB3 [23]. More importantly, lncRNA-SNHG5 was found to facilitate NPC development by upregulating HMGB3, which has not been investigated in NPC. Combining these results, we consider that IncRNA-SNHG5 functions as a tumor promoter in NPC by regulating miR-1179/HMGB3 axis.

\section{Conclusion}

In conclusion, LncRNA-SNHG5 promoted NPC progression by sponging miR-1179 and upregulating HMGB3. It indicates that LncRNA-SNHG5/ miR-1179/ HMGB3 pathway may possess a potential power in NPC treatments. Nevertheless, we only initially explained the regulatory mechanism of LncRNA-SNHG5 in NPC. The regulation network of IncRNAs is complex, and we still need to further explore the functional mechanism of SNHG5 in NPC.

\section{Supplementary information}

Supplementary information accompanies this paper at https://doi.org/10. 1186/s12885-020-6662-5.

\section{Additional file 1. \\ Additional file 2. \\ Additional file 3.}

Abbreviations

CCK-8: Cell counting kit-8; CDNA: Complementary DNA; HMGB3: High mobility group box 3; IncRNA: long non-coding RNA; miRNA: microRNA; mRNA: messenger-RNA; Mut: Mutant type; NPC: Nasopharyngeal carcinoma; RT-qPCR: Real-time quantitative PCR; SNHG5: Small Nucleolar RNA Host Gene 5; Wt: Wide type

\section{Acknowledgements}

Sincerely thanks all the participants for their support.

\section{Authors' contributions}

$\mathrm{DL}$ contributed to the data-analysis and revision of the manuscript. YW was involved in data acquisition and revision of the manuscript. YZ contributed to the study design, data analysis and drafted the manuscript. XG contributed to data analysis, revision of the manuscript and the conception and design of the study. All authors have read and approved the final manuscript

Funding

None.

Availability of data and materials

The datasets used and/or analyzed during the present study are available from the corresponding author on reasonable request.

Ethics approval and consent to participate

The study was approved by the Institutional Ethics Committee of Linyi People's Hospital. All the patients provided written informed consent.

Consent for publication

Not applicable.

Competing interests

All authors declare that they have no competing interests.

\section{Author details}

${ }^{1}$ Clinical laboratory, Linyi People's Hospital, Linyi, Shandong Province, People's Republic of China. 'E.N.T. Department, Linyi People's Hospital, No. 27 East section of Jiefang Road, Linyi 276000, Shandong Province, People's Republic of China.

Received: 25 July 2019 Accepted: 20 February 2020

Published online: 04 March 2020

References

1. Wei KR, Zheng RS, Zhang SW, Liang ZH, Li ZM, Chen WQ. Nasopharyngeal carcinoma incidence and mortality in China, 2013. Chinese J Cancer. 2017 36(1):90. 
2. Katano A, Takahashi W, Yamashita H, Yamamoto K, Ando M, Yoshida M, Saito Y, Abe O, Nakagawa K. Radiotherapy alone and with concurrent chemotherapy for nasopharyngeal carcinoma: a retrospective study. Medicine. 2018;97(18):e0502.

3. Bruce JP, Yip K, Bratman SV, Ito E, Liu FF. Nasopharyngeal Cancer: molecular landscape. J Clin Oncol. 2015;33(29):3346-55.

4. Zhang E, Li X. LnCRNA SOX2-OT regulates proliferation and metastasis of nasopharyngeal carcinoma cells through miR-146b-5p/HNRNPA2B1 pathway. J Cell Biochem. 2019; 120(10):1-14.

5. Chen L, Sun L, Dong L, Cui P, Xia Z, Li C, Zhu Y. The role of long noncoding RNA-LET in cell proliferation and invasion of nasopharyngeal carcinoma and its mechanism. OncoTargets Ther. 2017;10:2769-78.

6. Gao J, Zeng K, Liu Y, Gao L, Liu L. LncRNA SNHG5 promotes growth and invasion in melanoma by regulating the miR-26a-5p/TRPC3 pathway. OncoTargets Rher. 2019;12:169-79.

7. Hu X, Hong Y, Shang C. Knockdown of long non-coding RNA SNHG5 inhibits malignant cellular phenotypes of glioma via Wnt/CTNNB1 signaling pathway. J Cancer. 2019;10(5):1333-40.

8. Zhao L, Guo H, Zhou B, Feng J, Li Y, Han T, Liu L, Li L, Zhang S, Liu Y, et al. Long non-coding RNA SNHG5 suppresses gastric cancer progression by trapping MTA2 in the cytosol. Oncogene. 2016;35(44):5770-80.

9. Yoon JH, Abdelmohsen K, Gorospe M. Functional interactions among microRNAs and long noncoding RNAs. Semin Cell Dev Biol. 2014;34:9-14.

10. Jiang $L$, Wang $Y$, Rong $Y, X u L$, Chu Y, Zhang Y, Yao Y. miR-1179 promotes cell invasion through SLIT2/ROBO1 axis in esophageal squamous cell carcinoma. Int J Clin Exp Pathol. 2015;8(1):319-27.

11. Lin C, Hu Z, Yuan G, Su H, Zeng Y, Guo Z, Zhong F, Jiang K, He S. MicroRNA-1179 inhibits the proliferation, migration and invasion of human pancreatic cancer cells by targeting E2F5. Chem Biol Interact. 2018;291:6571.

12. Guo $S$, Wang $Y$, Gao $Y$, Zhang $Y$, Chen $M, X u M$, Hu L, Jing $Y$, Jing $F$, Li C, et al. Knockdown of high mobility group-box 3 (HMGB3) expression inhibits proliferation, reduces migration, and affects Chemosensitivity in gastric Cancer cells. Med Sci Monit. 2016;22:3951-60.

13. Li M, Cai Y, Zhao H, Xu Z, Sun Q, Luo M, Gu L, Meng M, Han X, Sun H. Overexpression of HMGB3 protein promotes cell proliferation, migration and is associated with poor prognosis in urinary bladder cancer patients. Tumour Biol. 2015;36(6):4785-92.

14. Yamada Y, Nishikawa R, Kato M, Okato A, Arai T, Kojima S, Yamazaki K, Naya $Y$, Ichikawa T, Seki N. Regulation of HMGB3 by antitumor miR-205-5p inhibits cancer cell aggressiveness and is involved in prostate cancer pathogenesis. J Hum Genet. 2018;63(2):195-205.

15. Kong YG, Cui M, Chen SM, Xu Y, Xu Y, Tao ZZ. LncRNA-LINC00460 facilitates nasopharyngeal carcinoma tumorigenesis through sponging miR-149-5p to up-regulate IL6. Gene. 2018;639:77-84.

16. Li J, Sun CK. Long noncoding RNA SNHG5 is up-regulated and serves as a potential prognostic biomarker in acute myeloid leukemia. Eur Rev Med Pharmacol Sci. 2018;22(11):3342-7.

17. Ju C, Zhou R, Sun J, Zhang F, Tang X, Chen KK, Zhao J, Lan X, Lin S, Zhang $Z$, et al. LncRNA SNHG5 promotes the progression of osteosarcoma by sponging the miR-212-3p/SGK3 axis. Cancer Cell Int. 2018;18:141.

18. Zhang M, Li Y, Wang H, Yu W, Lin S, Guo J. LncRNA SNHG5 affects cell proliferation, metastasis and migration of colorectal cancer through regulating miR-132-3p/CREB5. Cancer Biol Ther. 2019;20(4):524-36.

19. Xu X, Cai N, Zhi T, Bao Z, Wang D, Liu Y, Jiang K, Fan L, Ji J, Liu N. MicroRNA-1179 inhibits glioblastoma cell proliferation and cell cycle progression via directly targeting E2F transcription factor 5. Am J Cancer Res. 2017;7(8):1680-92.

20. Song L, Dai Z, Zhang S, Zhang H, Liu C, Ma X, Liu D, Zan Y, Yin X MicroRNA-1179 suppresses cell growth and invasion by targeting spermassociated antigen 5-mediated Akt signaling in human non-small cell lung cancer. Biochem Biophys Res Commun. 2018;504(1):164-70.

21. Gao J, Zou Z, Gao J, Zhang H, Lin Z, Zhang Y, Luo X, Liu C, Xie J, Cai C. Increased expression of HMGB3: a novel independent prognostic marker of worse outcome in patients with esophageal squamous cell carcinoma. Int J Clin Exp Pathol. 2015;8(1):345-52.

22. Liu J, Wang L, Li X. HMGB3 promotes the proliferation and metastasis of glioblastoma and is negatively regulated by miR-200b-3p and miR-200c-3p. Cell Biochem Funct. 2018;36(7):357-65.

23. Zhou GH, Lu YY, Xie JL, Gao ZK, Wu XB, Yao WS, Gu WG. Overexpression of miR-758 inhibited proliferation, migration, invasion, and promoted apoptosis of non-small cell lung cancer cells by negatively regulating HMGB. Biosci Rep. 2019;39(1):1-31.

\section{Publisher's Note}

Springer Nature remains neutral with regard to jurisdictional claims in published maps and institutional affiliations.
Ready to submit your research? Choose BMC and benefit from:

- fast, convenient online submission

- thorough peer review by experienced researchers in your field

- rapid publication on acceptance

- support for research data, including large and complex data types

- gold Open Access which fosters wider collaboration and increased citations

- maximum visibility for your research: over $100 \mathrm{M}$ website views per year

At BMC, research is always in progress.

Learn more biomedcentral.com/submissions 